\title{
Microemulsion Polymerization of Alkyl (Meth)acrylates Initiated by UV Light
}

\author{
Ignác CAPEK
}

\author{
Polymer Institute, Slovak Academy of Sciences, Dúbravská cesta \\ 9, 84236 Bratislava, Slovak Republic
}

(Received March 12, 1999)

\begin{abstract}
Radical polymerizations of polar unsaturated monomers ethyl acrylate, methyl methacrylate and nonyl methacrylate in an o/w microemulsion photoinitiated by a UV light were investigated. Polymerizations were conducted at low temperature to follow the effects of a varied solubility of monomer (coemulsifier) and/or diffusional degradation of monomer droplets on the kinetic and colloidal parameters. The rate versus conversion curve showed four regions with two rate maxima ca. at $5 \%$ to $20 \%$ and 50 to $70 \%$ conversion. The first rate maximum results from abrupt decrease of the monomer concentration at microemulsion loci and the second one from the increased accumulation of radicals in the latex particles. The higher polymerization rate of ethyl acrylate compared to that for methyl methacrylate or nonyl methacrylate was attributed to higher monomer concentration at reaction loci and propagation rate constant and lower quenching rate. The rate of polymerization was found to increase with incident light intensity and was most pronounced with nonyl methacrylate. The reaction order (the dependence of the rate of polymerization or the number of particles on the incident light intensity) was taken as a measure of particle nucleation efficiency. Particle size distribution was observed to be very narrow at low conversion and increased with increasing conversion.

KEY WORDS Microemulsion / Polymerization / Alkyl (Meth)acrylates / Kinetics / Particle / Nucleation /
\end{abstract}

Microemulsion and miniemulsion polymerizations offer convenient access to well-defined microlatex particles being typically one order of magnitude smaller than polymer particles obtained by conventional emulsion polymerization. The fine monomer emulsion exhibits a large interface between monomer droplets and the aqueous phase. Under such conditions, continuous nucleation up to (very) high conversion is operative. There is no apparent constant rate period in the $\mathrm{o} / \mathrm{w}$ microemulsion or miniemulsion polymerization. ${ }^{1-3}$ Ugelstadt et al. ${ }^{4}$ clearly demonstrated that monomer droplets could effectively compete with monomer-swollen micelles for capture of free radicals generated in the aqueous phase when the total surface area of the droplets became large enough. In conventional emulsion polymerization, the principle locus of particle nucleation is the aqueous phase or the monomer swollen micelles depending on the degree of water solubility of the monomers, an initiator and amount of emulsifier. Monomer droplets are considered only as monomer reservoir supplying monomer to the growing polymer particles. However, the small size of the monomer droplets (micro- or minidroplets) enables them to become the principle locus of particle nucleation.

The principle behind the formation of microemulsions (droplet size $10-50 \mathrm{~nm}$ ) is penetration of coemulsifier into the water/oil interface, thereby decreasing surface tension and increasing interface area. Coemulsifier also decreases the rigidity of interface film due to increasing the molecular disorder. Coemulsifier promotes the formation of a more curved interfacial area and this is reflected in smaller size of oil droplets. The coemulsifier (short alkyl chain alcohols) molecules penetrate the interface. If the elastic constant of the interface is of the order of the thermal energy, the interfacial film is flexible. Otherwise, the film becomes rigid (if the elastic constant is much larger than the thermal energy). Once the conditions are right, spontaneous formation of the microemulsion occurs and little mechanical work is re- quired. In classical microemulsion polymerization, the principal locus of particle nucleation is the emulsified monomer droplets (microdroplets), particle nucleation proceeds throughout the course of polymerization and the dependence of the rate $v s$. conversion is described by a curve with the one maximum at $c a .20-40 \%$ conversion.

Fine emulsions with larger droplet size (above $50 \mathrm{~nm}$ in diameter) are termed as miniemulsions. Miniemulsions are relatively stable submicron dispersions of monomer in water prepared by shearing a system containing monomer, water, emulsifier and hydrophobe. The principle behind the making of stable miniemulsions is the introduction of a hydrophobic component (hexadecane, polystyrene (PSt), poly(methyl methacrylate) (PMMA), etc.) into monomer droplets to retard substantially the diffusion of monomer out of the monomer droplets. ${ }^{3,5}$ In miniemulsion polymerization, particle nucleation is somewhat reduced up to $c a .30-50 \%$ and the dependence of the rate $v s$. conversion is described by a curve with two maxima (four rate intervals). In classical emulsion polymerization the nucleation interval ends below 5 to $20 \%$ conversion.

Ethyl acrylate (EA) and methyl methacrylate (MMA) monomers act as poor coemulsifiers in the microemulsion polymerization (at moderate temperatures) and particle nucleation does not proceed above $50 \%$ conversion. ${ }^{6,7}$ This somewhat deviates from the kinetics of microemulsion polymerization. The water solubility of both monomers is inversely proportional to temperature. Thus, at a low temperature the penetration of monomer into the interfacial layer is lower as well as colloidal stability of microdroplets. This is expected to influence kinetic and colloidal parameters of emulsion polymerization. This is one of the primary subjects for the present study. Nonyl methacrylate (NMA) acts as a coemulsifier (contains a polar group) and hydrophobe (a low water solubility) as well. ${ }^{8}$ At low temperature, the hydrophobic feature of NMA is expected to be more operative. This 
is a second aim of the present study. Radical formation was performed by UV light.

\section{EXPERIMENTAL}

\section{Materials}

Commercially available EA, MMA, and NMA were purified by usual methods. ${ }^{7,9}$ The photoinitiator system consisting of dodecyl thioxantone (DTX) and methyldiethanoleamine (MDEA) (both from Fluka) was used. The emulsifier used was the reagent-grade sodium dodecyl sulfate (SDS) (Fluka). Twice-distilled water was used as the polymerization medium.

Polymerization Procedure. Batch polymerizations were run at $23^{\circ} \mathrm{C}$. In all runs the recipe comprises $100 \mathrm{~g}$ water, $20 \mathrm{~g}$ SDS, $2.5 \mathrm{~g}$ monomer, $0.0122 \mathrm{~g}$ DTX and $0.0383 \mathrm{~g}$ MDEA. Incident light intensity $\left(I_{\mathrm{o}} /\right.$ einstein $\left.\mathrm{dm}^{-3} \mathrm{~s}^{-1}\right)$ varied as given later.

Polymerization technique. Polymerization experiments were performed on an optical bench using monochromatic light of the wavelength of $\lambda=365 \mathrm{~nm}$. Equipment, measurement of radiation intensity and photopolymerization technique have been described elsewhere. ${ }^{10}$ Conversion of monomer was determined by dilatometric measurements (checked by gravimetry). The polymerization rate $\left(R_{\mathrm{p}} / \mathrm{mol} \mathrm{dm}^{-3} \mathrm{~s}^{-1}\right)$ at different conversion was determined by a non-linear least squares regression analysis $\left\{R_{\mathrm{p}}=(\mathrm{d} x / \mathrm{d} t) \times n_{\mathrm{M}}\right.$, where $x$ is the weight fraction of monomer to polymer and $n_{\mathrm{M}}$ is the initial number of moles of monomer per unit volume of the aqueous phase\}.

\section{Polymer and Latex Characterization}

The polymerization method and measurements of the average particle size and the apparent particle size distribution (APSD) (by a light scattering) were the same as described earlier. ${ }^{5,11}$ Emulsified monomer droplet number in the monomer emulsions was recorded by optical microscope. PSD of several latex samples was also measured by transmission electron microscopy. ${ }^{12,13}$

\section{RESULTS AND DISCUSSION}

\section{Submicron Droplets}

A slight mixing of monomer, water, emulsifier and coemulsifier leads to the formation of microemulsion. Submicron minidroplets are also generated by mixing monomer and water in a high-shear mixer in the presence of appropriate amounts of emulsifier and hydrophobe. By cooling, transparent fine emulsions are transformed to semitransparent or milky ones. In some cases a one-phase system transforms to a two-phase one. The size of submicron monomer droplets can also be varied by temperature. Turbidity of fine MMA, EA, or NMA emulsions was observed to increase with decreasing temperature from 70 to $10^{\circ} \mathrm{C}$ and the increase was more pronounced in runs with a lower emulsifier concentration. The diffusional degradation of monomer droplets is more favored at low temperature. To keep the polymerization system transparent or semitransparent an appropriate weight ratio monomer/emulsifier was used.

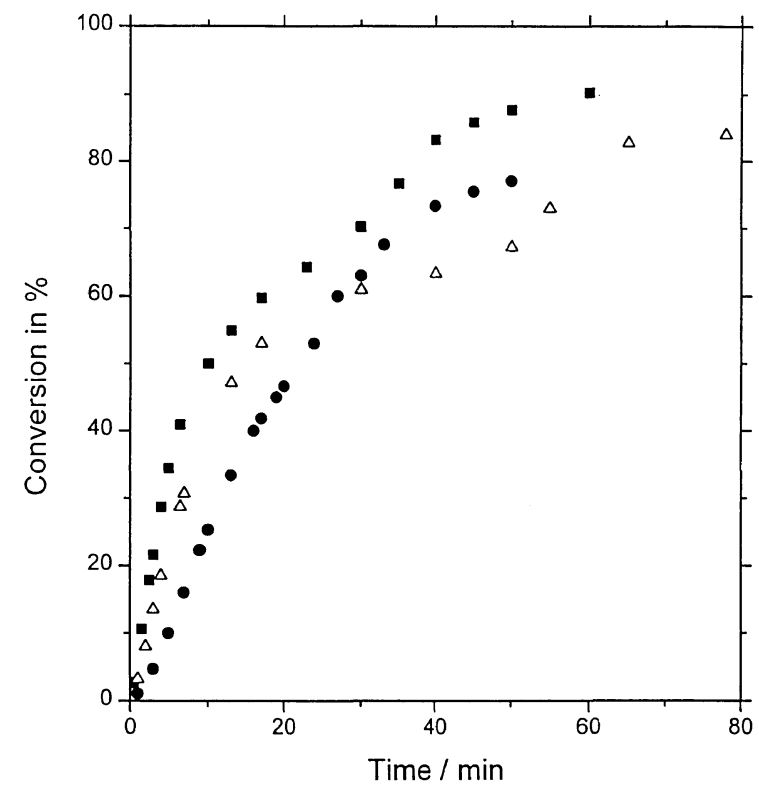

Figure 1. Variation of monomer conversion in microemulsion polymerization of EA $(\mathbf{\square})$, MMA $(\odot)$, and NMA $(\triangle)$ photoinitiated by a UV light with the reaction time. Recipe: $100 \mathrm{~g}$ water, $20 \mathrm{~g}$ SDS, $2.5 \mathrm{~g}$ monomer, $0.0122 \mathrm{~g}$ DTX, and $0.0383 \mathrm{~g} \mathrm{MDEA}, 23^{\circ} \mathrm{C}$; $I_{\mathrm{o}}=8.92 \times 10^{-6}$ einstein $\mathrm{dm}^{-3} \mathrm{~s}^{-1}$.

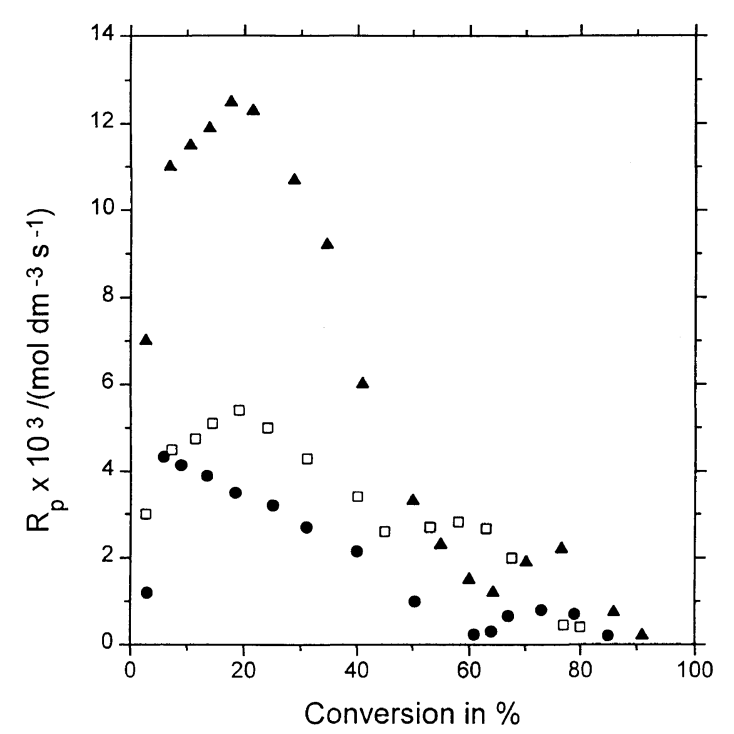

Figure 2. Variation of the rate of polymerization in the microemulsion polymerization of EA ( $\mathbf{\Delta})$, MMA ( $\square)$, and NMA (O) photoinitiated by the UV light with conversion. Other conditions see in the legend for Figure 1.

\section{Kinetic Parameters}

Conversion (X)-time data of the microemulsion polymerization of EA, MMA, and NMA initiated by the UV light $(\lambda=365 \mathrm{~nm})$ are shown in Figure 1 . All curves were found to have the same kinetic features. Corresponding polymerization rate $\left(R_{\mathrm{p}}\right)$ data are shown in Figure 2. This figure shows two rate maxima or four distinct nonstationary rate intervals. The results indicate that the polymerization rates of all runs exhibit similar behavior with respect to conversion, i.e., the polymerization rate first increases to a maximum followed by a decrease and then again increases to a second rate maximum followed by a final decrease. There is no 
significant constant rate interval observed. The first increase in the rate of polymerization is attributed to the intensive particle nucleation. ${ }^{6,7}$ Particle number was observed to increase from $5.8 \times 10^{16} / \mathrm{dm}^{3}$ to $2.7 \times$ $10^{17} / \mathrm{dm}^{3}$ from $10 \%$ to $30 \%$ conversion (with MMA), respectively, and then reached plateau. The rate of polymerization $\left(R_{\mathrm{p}, \max , 1}\right)$ for EA and MMA was maximum at $20 \%$ conversion while that for NMA was observed at $c a .5 \%$ conversion. $R_{\mathrm{p}, \max , 1}$ results from decreasing monomer concentration at the reaction loci and increasing the number of particles with conversion. The monomer polymer weight ratio for the emulsion polymerization of butyl acrylate (BA) under monomer saturation conditions was determined to be $c a \cdot 1.7 .^{14}$ The monomer/polymer weight ratio for the microemulsion polymerization of $\mathrm{BA}$ at $c a$. $40 \%$ conversion was below $1 .{ }^{15}$ Thus, the microemulsion polymerization of BA proceeds under monomer-starved conditions already present at medium conversion. Simulation of microemulsion polymerization of styrene led to the conclusion that the microdroplet monomer core disappears at conversion much below $10 \%$ conversion. ${ }^{1}$ The polymerization rate reaches maximum prior to the end of the nucleation period if the rate of formation of polymer particles slows down enough increase in the monomer concentration in the active latex particles. Under such conditions, decreasing monomer concentration in the particles dominates the rate of polymerization.

The different locations of $R_{\mathrm{p}, \max , 1}$ for NMA and EA (or MMA) result from the different lengths of monomer saturation interval. The monomer saturation interval is the shortest with NMA. This results from the hydrophobic nature of NMA, which decreases the rate of monomer diffusion to the reaction $\operatorname{loci}^{8}$ and so the rate of polymerization. The addition of a hydrophobic compound is known to decrease Ostwald ripening. ${ }^{3,5}$ The locations of hydrophobic molecules in the monomer droplets (highly monomer swollen particles) are supposed to depress diffusion of monomer from such monomer droplets to the active polymer particles. Under such conditions, the rate polymerization of NMA decreases nearly from the start of polymerization. A large number of fine emulsified monomer droplets $\left(\mathrm{ca} \cdot 10^{21}\right.$ per $\mathrm{dm}^{3}$ ) promotes the continuous nucleation of polymer particles up to high (final) conversion. The concentration of monomer decreases throughout the polymerization, ${ }^{1,15}$ and that monomer droplets exist throughout the polymerization. ${ }^{16}$ Droplet size measurements did not confirm the presence of monomer droplets above $300-500 \mathrm{~nm}$ in diameter (smallest droplets which could be measured by optical microscopy) during the whole polymerization. This indicates the presence of tiny monomer droplets in the reaction system. The monomer droplets (with diameter above $300-500 \mathrm{~nm}$ ), however, were observed in the fine emulsion polymerization of styrene up to $80-90 \%$ conversion. ${ }^{13,17} R_{\mathrm{p}} v s$. conversion curve showed one distinct maximum at $c a$. $20-40 \%$ and a second rate shoulder at $c a .70-80 \%$ conversion. The second rate maximum was accompanied by increase in the average number of radical per particle (the gel effect).

The second rate maximum did not appear in the microemulsion polymerization ${ }^{1,2,6,7,18,19}$ but in the miniemulsion polymerization..$^{3,5,20}$ Two rate maxima were reported in the classical miniemulsion polymerizations of styrene or MMA in the presence of the strong hydrophobe (cetyl alcohol (CA), hexadecane (HD), etc.) and/or poor hydrophobe (PMMA or PSt). The first rate maximum was more pronounced with PSt (or PMMA) while the second maximum was more pronounced with CA (or HD). The shape of $R_{\mathrm{p}} v s$. conversion curve for EA, MMA, or NMA is more like that observed in the miniemulsion polymerization of styrene or MMA in the presence of a poor hydrophobe (PMMA or PSt). ${ }^{5}$ The distinct second maximum, however, was expected with NMA as an efficient hydrophobe. ${ }^{12}$ The results show that this is not the case. This behavior can be attributed to the location of NMA in the interfacial layer.

The first $\left(R_{\mathrm{p}, \max , 1}\right)$ and second rate $\left(R_{\mathrm{p}, \max , 2}\right)$ maxima decrease as follows:

$$
\begin{aligned}
& R_{\mathrm{p}, \text { max }, 1}: \mathrm{EA}>\mathrm{MMA} \geq \mathrm{NMA} \\
& \quad \text { and } \quad R_{\mathrm{p}, \max , 2}: \mathrm{MMA}>\mathrm{EA}>\mathrm{NMA}
\end{aligned}
$$

The ratio $R_{\mathrm{p}, \max , 1} / R_{\mathrm{p}, \max , 2}\left(R_{1 / 2}\right)$ varies as follows:

$$
5.9(\mathrm{EA})>5.5(\mathrm{NMA})>1.9(\mathrm{MMA})
$$

The largest $R_{\mathrm{p}, \max , 1}$ or $R_{1 / 2}$ for EA can be attributed to high propagation rate constant (ca. one order in magnitude larger than that for MMA or NMA $\left.{ }^{19,21}\right)$ and high monomer concentration at reaction loci (monomer only partly penetrates the interface). The main location of NMA at the interfacial layer decreases the monomer concentration at the reaction loci (dilution effect) ${ }^{22}$ and the rate of polymerization as well. The largest $R_{\mathrm{p}, \max , 2}$ or smallest $R_{1 / 2}$ for MMA results from increased agglomeration of particles which first decreases $R_{\mathrm{p}, \max , 1}$ (due to increased deactivation of reaction loci) and later increases $R_{\mathrm{p}, \max , 2}$ (due to accumulation of radicals in the large particles)

The high emulsifier/water ratio ensures that the largest fraction of emulsifier is undissociated. This is a basic rule for the formation of microemulsions. ${ }^{23}$ The degree of (emulsifier) dissociation is inversely proportional to temperature. Under the current reaction conditions, solubility of the emulsifier in the monomer phase is relatively high. ${ }^{24}$ The monomer-soluble emulsifier decreases the average monomer concentration at the reaction loci and promotes the chain transfer to emulsifier. $^{25}$ The accumulation of emulsifier in the monomer droplets is expected to decrease the rate of polymerization with conversion. The presence of hydrophobic (undissociated) emulsifier in the microdroplets can disfavor Ostwald ripening.

The polymerization rate continuous to increase to about $60 \%$ for MMA and $75 \%$ for EA and NMA when particles reach their glass transition point, bringing about a reduction in the propagation rate and thus reducing the polymerization rate. This causes decrease in the rate of termination inside the monomer-swollen particle. The observed behavior is very similar to that reported by El-Aasser et al. ${ }^{20}$ and Shork et al. ${ }^{5}$ for the classical miniemulsion polymerizations of styrene and MMA in the presence of hydrophobes such as CA, $\mathrm{HD}$, polymer, etc. and homogenized by a uniform shear device (Microfluidizer). 
$R_{\mathrm{p}, \max , 1}$ varies with the incident light intensity, $I_{\text {inc }}$, as follows ( $c f$. Table I):

$$
R_{\mathrm{p}, \text { max }, 1} \propto I_{\text {inc,EA }}^{0.59}, R_{\mathrm{p}, \max , 1} \propto I_{\text {inc,MMA }}^{0.53} \text { and } R_{\mathrm{p}, \text { max }, 1} \propto I_{\text {inc,NMA }}^{0.77}
$$

The largest reaction order $x\left(R_{\mathrm{p}, \max , 1} \times I_{\text {inc }}^{x}\right)$ is found with NMA. Less pronounced increase in $R_{\mathrm{p}, \max , 1}$ is found with EA or MMA. In the thermally (peroxodisulfate) initiated polymerization of alkyl (meth)acrylates, the reaction order $x$ is much smaller than 0.7 . The similar reaction order $x$, however, was observed in the photoinitiated microemulsion polymerization of butyl acrylate. ${ }^{18,19}$ This was attributed to the high entry efficiency of radicals (without charge) formed during photolysis. The interaction between monomer and emulsifier leads to the formation of additional radicals increasing the flux of radicals into the microdroplets. In the photoinitiated microemulsion polymerization of acrylamide, the rate of polymerization was found proportional to the first power of incident light intensity and $\alpha, \alpha$-azobis(isobutyronitrile) (AIBN) concentration. ${ }^{26}$ The rate of classical emulsion polymerization slightly increase with AIBN concentration (reaction order is much below 0.4$).{ }^{27}$ In the microemulsion polymerization of acrylamide, ${ }^{26}$ the diffusion of a water soluble monomer through the oil phase (toluene, decane, etc.) is strongly depressed while in the emulsion polymerization of styrene ${ }^{27}$ the monomer droplets act as a monomer reservoir, the diffusion of monomer is unrestricted, the diffusional degradation of monomer droplets is operative, the initiating radicals are mainly captured by the polymer particles and nucleation of particles during the interval 2 (stationary interval) is negligible.

Thus, variation of the reaction order $x$ with the polymerization mode (microemulsion (Micr.) or emulsion (Emul.)) can be summarized as follows:

1.0 (acrylamide, Micr. ${ }^{26}$ ) $>0.87$ (Styrene, Micr. ${ }^{28}$ ) $>0.77$ (NMA) $>0.7$ (butyl acrylate, Micr. ${ }^{18,19}$ ) $>0.59$ (EA) $>$ 0.53 (MMA) >0.37 (Styrene, Emul., ${ }^{28}$ ).

The reaction order $x$ can also be discussed in terms of particle nucleation. The smallest reaction order $(x=0.37)$ was found in the emulsion polymerization of styrene where the nucleation period is very short and the bimolecular termination is operative in the aqueous phase and latex particles. A somewhat larger reaction order $(x=0.53$ and 0.59$)$ was observed in microemulsion polymerizations of MMA and EA where the spontaneous particle nucleation was prolonged up to 30 or $40 \%$ conversion. Above this conversion, the number of particles depressed. ${ }^{6,7}$ Particle growth was attributed to propagation of monomer and agglomeration of latex particles. In the microemulsion polymerization of hydrophobic BA and NMA monomers the reaction order is 0.7 and 0.77 , respectively. Here the particle nucleation proceeded up to ca. 90 to $100 \%$ conversion. The continuous nucleation up to the final conversion (with the reaction order $x=1$ ) was observed in the microemulsion polymerization of acrylamide.

In the peroxodisulfate-initiated microemulsion polymerization of styrene continuous nucleation up to very high conversion and large reaction order $x=0.87$ were reported. ${ }^{28}$ In this case, the released coemulsifier from polymer particles (pentanol is not compatible with PSt) is assumed to penetrate the surface layer of monomerswollen micelles due to which the surface area of free microdroplets is $c a$. constant (or increases). Consequently, radical entry efficiency for micelles is very high, as well as nucleation activity. Acrylamide monomer microemulsions were found to be highly percolating and the percolation activity decreased with increasing conversion (decreasing acrylamide (coemulsifier) concentration). ${ }^{29}$ The formation of transient (percolating) aggregates is most pronounced at low conversion. Under such conditions, the active surface area for radical entry is lowered. As a polymerization advances, the percolating aggregates decay to single droplets due to which the surface area of non-nucleated micelles remains $c a$. constant (or increases). Consequently, the particle nucleation increases and the reaction order $x$ is close to 1 .

The reaction order 0.5 refers to the bimolecular termination for the bulk or pseudo-bulk (radicals do not sense the interface zone) systems and 1.0 to the monomolecular termination caused by degradative chain transfer, occlusion of growing radicals, etc. In micellar systems, reaction loci are isolated and termination proceeds by chain transfer to monomer, emulsifier and additive. In fine emulsion systems, transferred radicals desorb to the aqueous phase and then re-enter microdroplets. Chain transfer/desorption/re-entry events govern the reaction mechanism. This results from the much large surface area ( 2 or 3 orders of magnitude) provided by microdroplets compared to that provided by the latex particles. Thus, radical flux to the microdroplets is large while to polymer particles is very small. Besides, the surface area of microdroplets is a complex function of formation or decay of percolating aggregates, the barrier for entering radicals and the degree of monomer solubility of emulsifier. Monomer swollen inactive polymer particles mainly serve as a reservoir of monomer and emulsifier.

\section{Colloidal Parameters}

Table I shows that the particle size $(D / \mathrm{nm})$ and the particle size distribution (APSD or PSD) were observed to increase in the following order (Table I, run 3):

$$
D_{\mathrm{n}}: 25 \mathrm{~nm}(\mathrm{NMA})<28 \mathrm{~nm}(\mathrm{EA})<47 \mathrm{~nm}(\mathrm{MMA})
$$

and

$$
\begin{aligned}
& \text { APSD }(>80 \% \text { conversion }): \\
& 1.2(\mathrm{NMA})<1.5(\mathrm{EA})<2.4(\mathrm{MMA}) \\
& \text { APSD }(\leq 20 \% \text { conversion }): \\
& 1.05(\mathrm{NMA})<1.09(\mathrm{EA})<1.18(\mathrm{MMA}) \\
& \text { PSD }(>80 \% \text { conversion }): \\
& 1.05(\mathrm{NMA})<1.2(\mathrm{EA})<1.6(\mathrm{MMA})
\end{aligned}
$$

Particle size decreases with increasing the alkyl group of (meth)acrylate monomer. The larger coemulsifier activity the smaller particle size. The microemulsion polymerization is known to produce the monodisperse polymer latexes. The experimental data indicate that APSD or PSD increases with conversion and the increase is much more pronounced for APSD (light scattering estimates). The latex particles with narrow distribution are formed at low conversion. Thus, the reaction system is ther- 
Table I. Variation of kinetic parameters in the microemulsion polymerization of EA, MMA, and NMA with the incident light intensity

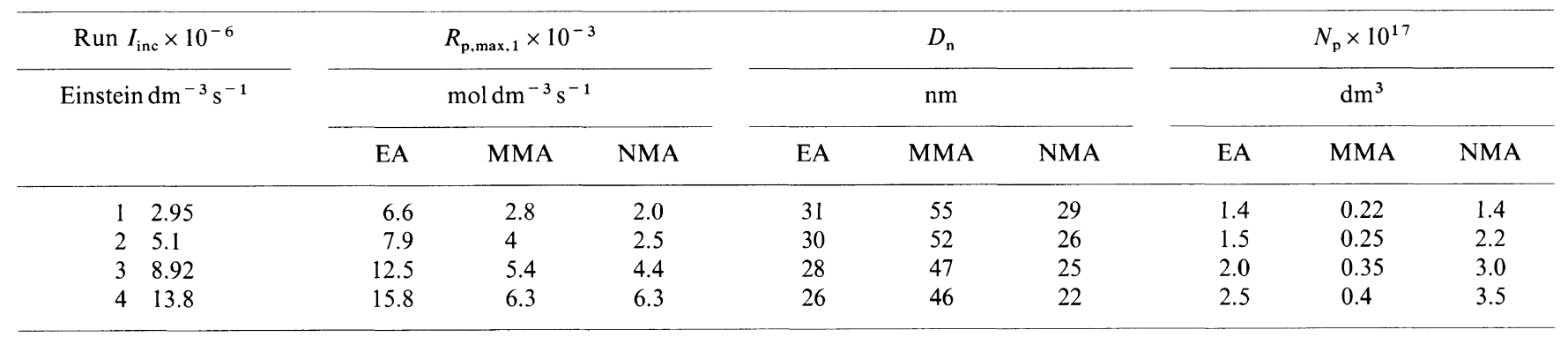

${ }^{\text {a }}$ Final conversion varied from 80 to $100 \%$.

modynamically stable without or with a low level of polymer. The accumulation of polymer in the system depresses the colloidal stability of reaction system, connected to the increase of PSD. The increase of PSD from NMA to MMA results from lower coemulsifier activity of MMA or lower colloidal stability (increased diffusional degradation) of MMA microemulsion. The location of NMA molecules in the interface layer increases the interface area of submicron droplets, the number of microdroplets (latex particles) and formation of monodisperse polymer latexes. The hydrophobic NMA increases the colloidal stability of microdroplets.

The number of particles was found to increase with incident light intensity $\left(N_{\mathrm{p}} \propto I_{\text {inc }}^{y}\right)$, according to ( $c f$. Table I):

$$
N_{p} \propto I_{\text {inc,EA }}^{0.4}, N_{\mathrm{p}} \propto I_{\text {inc,MMA }}^{0.38} \quad \text { and } \quad N_{\text {p }} \propto I_{\text {inc,NMA }}^{0.6}
$$

Nucleation activity increases from MMA, EA to NMA. This is in good agreement with the kinetic approach discussed above. However, the (colloidal) reaction order $(y)$ is lower than the (kinetic, $R_{\mathrm{p}, \max } v s$. $I_{\text {inc }}^{x}$ ) reaction order $(x)$. This difference is attributed to variation of nucleation activity with conversion. The kinetic order $(x)$ was estimated at $c a .20 \%$ conversion (high nucleation activity) while the colloidal order $(y)$ was estimated at $c a$. $80 \%$ (the decreased nucleation activity). Quite different behavior was observed in the peroxodisulfateinitiated microemulsion polymerization of alkyl (meth)acrylates where the reaction order $y$ is close to zero or reaches a negative value. ${ }^{18,19}$ The high particle nucleation activity of the current systems may result from the high level of undissociated emulsifier in the present system or a high monomer solubility of emulsifier. The more close packed structure of the interfacial layer formed at lower temperature promotes higher colloidal stability and nucleation efficiency of microemulsion. The reversed trend is found in the microemulsion polymerization of styrene where the (colloidal) reaction order $(0.59)$ is larger than the kinetic one (0.4), respectively. ${ }^{30}$ This can be discussed in terms of varied level of pentanol in the interface during polymerization. The released coemulsifier (due to restricted compatibility with polymer) penetrates into the interfacial layer and so increases the surface area of the microdroplets.

\section{Photophysics of Radical Formation}

The quantum yield $\phi_{\mathrm{m}}$ of polymerization initiated by DTX/MDEA was estimated as $c a .1400 .{ }^{31}$ This indicates that the initiating efficiency of the DTX/MDEA system is very high. In the absence of a cosynergist (MDEA) the rate of polymerization is in one or two orders in magnitude lower. The thioxanthone triplet was observed to be strongly quenched by alkyl methacrylates and the quenching rate constant for triplet thioxantones by methyl methacrylate undergoes a drastic decrease when the solvent polarity increases. ${ }^{32}$ The location of hydrophobic DTX in the monomer droplets indicates that the triplet DTX might be partially quenched by MMA or NMA. This might decrease the overall rate of polymerization in MMA and NMA runs. The low quenching rate for EA increases the concentration of initiating radicals via interaction of triplet DTX and MDEA and the rate of polymerization as well.

\section{CONCLUSIONS}

The rate versus conversion curve showed four regions with two rate maxima $c a$. at $5 \%$ to $20 \%$ and 50 to $70 \%$ conversion. The first rate maximum on the rate-conversion curve is a basic characteristic of a microemulsion system as reported in several papers, ${ }^{1,2,5,6}$ due to; 1) increase of particle concentration and 2) decrease of the monomer concentration at the reaction loci with increasing conversion. The second rate results from accumulation of radicals in the latex particles due to increased particle size. The larger rate of polymerization rate for EA compared to that for MMA or NMA is discussed in terms of the different monomer concentration at the reaction loci, number of particles, and propagation rate constant. The rate of polymerization was found to increase with incident light intensity and the increase was most pronounced in the NMA system. The high radical rate formation promotes the formation of fine polymer dispersion. This results from the close packed structure of the interfacial layer and high entry efficiency of radicals formed during photolysis. The finest dispersion was formed with NMA while the more course one with MMA. The same was noted for PSD.

Acknowledgments. This research was supported by the Slovak Grant Agency (VEGA) through grant numbers $2 / 5005 / 98$. The author thanks Mrs. O. Jurikova for assistance in the experiments.

\section{REFERENCES}

1. J. S. Guo, E. D. Sudol, J. W. Vanderhoff, and M. S. Elaasser, $J$. Polym. Sci., Part A, Polym. Chem., 30, 691 (1992); ibid., 30, 703 (1992). 
2. P. Potisk and I. Capek, Angew. Makromol. Chem., 222, 125 (1994).

3. Y. T. Choi, M. S. El-Aasser, E. D. Sudol, and J. W. Vanderhof, J. Polym. Sci., Polym. Chem. Ed., 23, 2973 (1985).

4. J. Ugelstad, M. S. El-Aasser, and J. W. Vanderhoff, J. Polym. Sci., Polym. Lett. Ed., 111, 503 (1973).

5. J. L. Reimers and F. J. Schork, J. Appl. Polym. Sci., 60, 251 (1996).

6. I. Capek and V. Juranicova, Eur. Polym. J., 34, 783 (1998).

7. I. Capek, V. Juranicova, K. Ito, J. Barton, and J. M. Asua, Polym. Int., 43, 1 (1997).

8. C. S. Chern and T. J. Chen, Colloid Polym. Sci., 275, 546 (1997).

9. T. Tanrisever, O. Okay, and I. C. Sonmezoglu, J. Appl. Polym. Sci., 61, 485 (1996).

10. J. Barton, I. Capek, and P. Hrdlovic, J. Polym. Sci., Polym. Chem. Ed., 13, 2671 (1975).

11. I. Capek and W. Funke, Makromol. Chem., 191, 2549 (1990).

12. I. Capek, M. Riza, and M. Akashi, Makromol. Chem., 193, 2843 (1992).

13. C. S. Chern, S. Y. Lin, and T. J. Hsu, Polym. J., 31, 516 (1999).

14. I. Capek, J. Barton, and E. Orolinova, Chem. Zvesti, 38, 803 (1984).

15. I. Capek and P. Potisk, Eur. Polym. J., 31, 1269 (1995).

16. J. Delgado, M. S. El-Aasser, C. A. Silebi J. W. Vanderhoff, and J. Guilot, J. Polym. Sci., Part B, Polym. Phys., 26, 1495 (1988).

17. S. Y. Lin, I. Capek, T. J. Hsu, and C. S. Chern, Langmuir, submitted.
18. I. Capek and J. P. Fouassier, Eur. Polym. J., 33, 173 (1997)

19. I. Capek, Polym. Int., 40, 41 (1996).

20. P. L. Tang, E. D. Sudol, C. A. Silebi, and M. S. El-Aasser, J. Appl. Polym. Sci., 43, 1059 (1991).

21. J. Brandrup and E. H. Immergut, Ed., "Polymer Handbook," 3rd ed, Wiley, New York, N.Y., 1989.

22. I. Capek and P. Potisk, J. Polym. Sci., Part A, Polym. Chem., 33, 1675 (1995).

23. I. Capek, Adv. Colloid Interface Sci., 80, 85 (1999).

24. S. Y. Lin, I. Capek, T. J. Hsu, and C. S. Chern, to be published.

25. J. Barton, V. Juranicova, and V. Vaskova, Makromol. Chem., 186, 1935 (1985).

26. M. T. Carver, U. Dreyer, R. Knoesel, F. Candau, and R. M. Fitch, J. Polym. Sci., Part A, Polym. Chem., 27, 2161 (1989).

27. X. Xu, Z. Zhang, X. Ge, and M. Zhang, J.Polym. Sci., Part A, Polym. Chem., 36, 257 (1998).

28. J. Barton, Progr. Polym. Sci., 21, 399 (1996).

29. M. T. Carver, E. Hirsch, J. C. Wittmann, R. M. Fitch, and F. Candau, J. Phys. Chem., 93, 4867 (1989).

30. L. M. Gan, C. H. Chew, J. H. Lim, K. C. Lee, and L. H. Gan, Colloid Polym. Sci., 272, 1082 (1994).

31. D. J. Lougnot, C. Turck, and J. P. Fouassier, Macromolecules, 22, 108 (1989).

32. J. F. Fouassier, P. Jacques, and M. V. Encinas, Chem. Phys. Lett., 148, 309 (1988). 